\title{
Photobiomodulation Improves Memory in Mild Cognitive Impairment: Three Case Reports
}

\author{
Agnes S Chan ${ }^{1,2 *}$, TszLok Lee ${ }^{1}$, Sophia Sze ${ }^{2}$, Michael R Hamblin ${ }^{3}$ \\ ${ }^{1}$ Neuropsychology Laboratory, Department of Psychology, The Chinese University of Hong Kong, Hong Kong \\ SAR, China
}

${ }^{2}$ Research Center for Neuropsychological Well-Being, The Chinese University of Hong Kong, Hong Kong SAR, China

${ }^{3}$ Laser Research Centre, Faculty of Health Science, University of Johannesburg, Doornfontein 2028, South Africa

\begin{abstract}
Objective: To examine the effect of photobiomodulation (PBM) on cognitive performance in older adults with mild cognitive impairment (MCl).

Background: While some positive effects of PBM stimulation to improve cognitive function in patients with traumatic brain injury and dementia has been reported, the PBM effects on amnestic mild cognitive impairment are relatively unexplored.

Materials and methods: Three patients (mean age $=62$ ) received 18-sessions of PBM stimulation twice per week for nine weeks. Each patient was assessed with Rey- Osterrieth Complex Figure Test (Rey-O testing visual memory) and Hong Kong List Learning Test (HKLLT testing verbal memory), Clinical Dementia Rating Scale (CDR), Functional Activities Questionnaire (FAQ), Geriatric Anxiety Scale-10 Item Version (GAS-10) and Chinese Geriatric Depression Scale (CGDS) before and after PBM.
\end{abstract}

Results: All subjects demonstrated memory impairment at baseline, which was improved within the normal range after the intervention. One subject's verbal memory improved from $1^{\text {st }}$ percentile (moderately impaired) to $67^{\text {th }}$ percentile (average), another patient improved from $4^{\text {th }}$ percentile to $26^{\text {th }}$ percentile, and the third from $11^{\text {th }}$ percentile to $54^{\text {th }}$ percentile.

Conclusions: PBM treatment may be a promising noninvasive intervention for patients with $\mathrm{MCl}$ or other memory disorder types.

\section{Keywords}

Mild cognitive impairment, Amnestic, Memory improvement, Memory testing, Photoneuromodulation, Low-level light therapy

\section{Introduction}

Photobiomodulation (PBM) is a non-invasive technique that utilizes light energy with wavelengths in the visible (400-700 $\mathrm{nm})$ and/or near-infrared (750-1100 $\mathrm{nm})$ range to activate cellular activity [1]. It has been proposed that the effect of light energy on enhancing microcirculation and central hemodynamics of the brain constitutes the fundamental mechanism in improving cognitive abilities $[2,3]$. There has been a growing interest in applying PBM as a possible intervention for cognitive disorders, including traumatic brain injury (TBI) [4-8] and dementia related to Alzheimer's disease or Parkinson's disease [8-13]. A case report showed significantly improved executive functions and verbal learning and memory in two TBI patients after PBM [5]. Hipskind, et al. [4] also reported promising PBM effects on 12 chronic TBI patients, who showed significant improvement in neuropsychological tests after 6-weeks of intervention. PBM studies on dementia patients have also show nencouraging results. Saltmarche, et al. [9] reported significant improvement in general cognitive function, better sleep, and less anxiety after 12-weeks of PBM treatment in five demented patients. Chao's pilot trial reported significantly improved cognitive functions in four patients with dementia after 12-weeks of PBM [10]. Another study on 11 patients with dementia also improved executive functions after 28 consecutive daily, 6-minute PBM sessions [11].

*Corresponding author: Agnes S Chan, Department of Psychology, The Chinese University of Hong Kong Shatin, NT, Hong Kong SAR, Tel: 852-39436654

Accepted: June 11, 2021

Published online: June 14, 2021

Citation: Chan AS, Lee T, Sze S, et al. (2021) Photobiomodulation Improves Memory in Mild Cognitive Impairment: Three Case Reports. Alzheimers Dis Dement 5(1):126-131 
Despite the encouraging findings on TBI and dementia, PBM effects on amnestic mild cognitive impairment ( $\mathrm{MCl})$ are relatively less studied. Mild cognitive impairment $(\mathrm{MCl})$ is defined as a transitional stage between the loss of brain function caused by normal aging and the onset of dementia. It has been found that about $19 \%-50 \%$ of patients with an $\mathrm{MCl}$ diagnosis progress to dementia over three years. Our recent study showed that older adults with $\mathrm{MCl}$ after a single stimulation demonstrated improved memory function, and similar improvement was also observed in normal older adults [14]. In specific, only those who received the stimulation but not those who received sham stimulation showed improved inhibition ability and mental flexibility. The functional nearinfrared spectroscopy (fNIRS) results further showed that older adults who received PBM also reduced hemodynamic response when performing a difficult task, suggesting that the difficult task may become less effortful for the older adults [15].

For the past few years, our center has tested PBM on patients with cognitive disorders. The present study reported the improvements in three patients with the amnestic type of $\mathrm{MCl}$ after 9 weeks of intervention. The treatment results revealed in this case report may shed light on the potential efficacy and applicability of PBM as a possible intervention for patients with $\mathrm{MCl}$.

\section{Methods}

\section{Participants}

The subjects were classified with Amnesic $\mathrm{MCl}$ according to the criteria proposed by the National Institute of AgingAlzheimer's Association workgroups [16]. At the baseline, they had a negative history of dementia or other neurological disorders, diabetes, atherosclerosis, hypothyroidism, and cardiac disease. They were administered the Chinese version of Mattis Dementia Rating Scale [17] (CDRS), which is a validated screening for dementia, and all patients scored above the clinical cutoff in CDRS, which suggests no evidence for dementia. Written informed consent was obtained from each patient. Ethics approval was obtained from the Joint Chinese University of Hong Kong-New Territories East Cluster Clinical Research Ethics Committee.

Before and after the intervention, each patient was tested individually with standardized neuropsychological tests on cognitive functions, psychological measures on mood state and anxiety levels, and standardized questionnaires on daily functioning abilities by well-trained research assistants.

\section{Measures}

The assessment tools adopted are standardized and sensitive measures of general cognitive abilities, adaptive living abilities, verbal and visual memory functions, mood status, and anxiety level. Each patient's test performance was compared with the normative samples of their similar age and educational levels. The tests are as follows:

Clinical Dementia Rating Scale [18] (CDR): CDR is a wellestablished semi-structured interview that evaluates six cognitive and functional performance domains applicable to $\mathrm{MCl}$ and dementia.
Functional Activities Questionnaire [19] (FAQ): FAQ is a standardized questionnaire of instrumental activities of daily living and can differentiate $\mathrm{MCl}$ from dementia. It measures ten aspects of functional skills in everyday life, for example, preparing balanced meals, managing personal finances, and traveling around.

Hong Kong List Learning Test [20] (HKLLT): HKLLT is an ecologically validated verbal memory test that is commonly adopted in Hong Kong. It requires the examinee to learn a list of 16 two-character Chinese words three times and recall them from memory after 10 minutes and 30 minutes.

Rey-Osterrieth Complex Figure Test [21] (Rey-O): Rey-O is a standardized visual memory test that requires the examinee to reproduce a complicated line drawing and then draw from memory after 30 minutes.

Geriatric Anxiety Scale-10 Item Version [22] (GAS-10): GAS-10 is a brief self-report questionnaire measuring the degree of anxiety or stress-related symptoms, e.g., irritability, tiredness, muscle tension, restlessness.

Chinese Geriatric Depression Scale [23] (CGDS): CGDS is a standardized self-report questionnaire measuring the degree of depressive symptoms, e.g, life satisfaction, sense of uselessness, helplessness, sadness. We adopted the 15 -item short-form.

\section{PBM Stimulation}

PBM was applied using a Wisefori 5-3800 model ( Wisefor Ltd. Hong Kong). The device contained up to nine individual LED nodes, which can be placed at various points according to the international 10/10 system, and it emitted $810 \mathrm{~nm}$ light at an irradiance of $20 \mathrm{~mW} / \mathrm{cm}^{2}$. Using a patented design, the protocol could be adjusted using a smart phone. This device was granted a CE certificate and is registered by the FDA as a Class 1 device. The duration of stimulation was 350 seconds, which generated a fluence of $7 \mathrm{~J} / \mathrm{cm}^{2}$. There were altogether 18 sessions of PBM stimulation, twice per week for nine weeks. Each session lasted for around 20 minutes with three stimulation sections and a one-minute break in between.

\section{Case Presentation}

Case P1: P1 was a 48 years old female housewife with 11 years of education, and she had a negative history of neurological disorders. At the baseline, her age- and the education-adjusted total score (146.2) in the CDRS were above the clinical cutoff for dementia (Table 1). She scored $0.5 / 3$ in the standard global score of CDR and scored $6 / 30$ in the FAQ (a measure of daily living activities), suggesting that she had some difficulty and may require assistance in performing some daily living chores. Besides, she showed a mild level of anxiety problems (total score $=9$ ) as measured by the GAS-10 and a below the clinical cutoff level of depressive symptoms (total score $=3$ ) as measured by CGDS at the baseline (Table 1 ).

Her memory has improved significantly after the intervention, as indicated by her performance on the preand post- neuropsychological memory tests. Her baseline 30-minute delayed recall performance in the HKLLT (a verbal memory test) was at the $1^{\text {st }}$ percentile rank (-2.17SD; 
clinically classified in the moderately impaired range), and her performance on the Rey-O (a visual memory test) was at the $10^{\text {th }}$ percentile $(-1.27 \mathrm{SD}$; clinically classified as a borderline range). After the intervention, P1's memory function had improved significantly. Her verbal memory improved by $2.62 \mathrm{SD}$ (from $1^{\text {st }}$ percentile to $67^{\text {th }}$ percentile), her performance after the course of PBM stimulation was classified as average, while the baseline level was classified as moderately impaired. Her visual memory was also improved by $0.36 \mathrm{SD}$ (from $10^{\text {th }}$ percentile to $18^{\text {th }}$ percentile) (Figure 1). Before PBM stimulation, P1 commented that she would always be forgetful, but since the second month of PBM, she reported that she could recall more events than before.

Besides, her CDR global score dropped from 0.5 to 0 (Table 2). At baseline, she had reported severe memory loss and slight impairment in judgment and problem solving; however, she reported that these functions had improved at the postintervention assessment. Her FAQ total score dropped from 6 to 1 , and these results suggested that her daily functioning had improved from some to very minimal difficulty. She indicated mild anxiety at the baseline, which had improved to

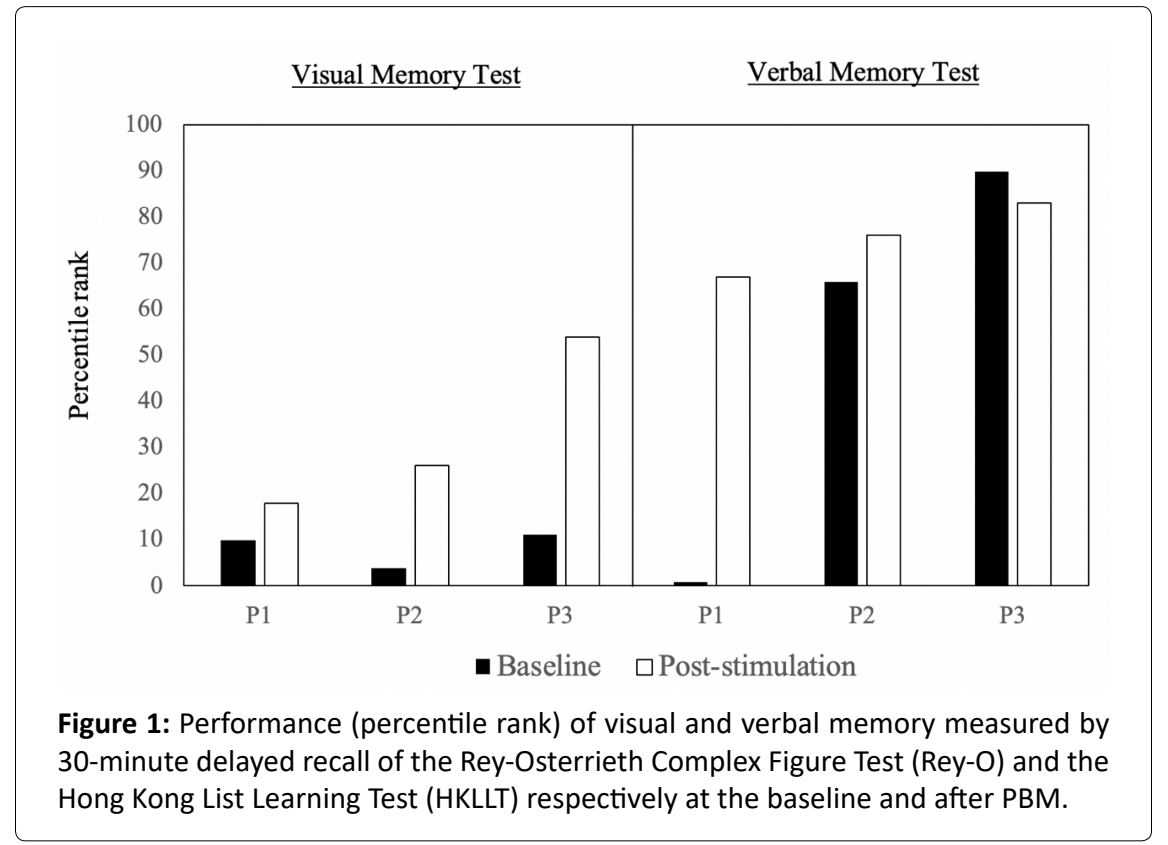

Table 1: Demographics and baseline characteristics of each participant.

\begin{tabular}{|c|c|c|c|c|c|c|c|}
\hline Participant & Age & Gender & Work Status & $\begin{array}{c}\text { Years of } \\
\text { Education }\end{array}$ & CDRS Score & CDR Score & FAQ Score \\
\hline P1 & 48 & F & Housewife & 11 & 146.20 & 0.5 & 6 \\
\hline P2 & 73 & F & Retired & 13 & 156.79 & 0.5 & 5 \\
\hline P3 & 66 & F & Retired & 11 & 158.29 & 0.5 & 2 \\
\hline Mean & 62.33 & & & 11.67 & 153.76 & 0.5 & 4.33 \\
\hline SD & 12.90 & & & 1.15 & 6.59 & 0 & 2.08 \\
\hline
\end{tabular}

CDRS Score $=$ Total score of Cantonese version of Mattis Dementia Rating Scale adjusted by the participant's age and education level; CDR Score $=$ Standard global score of the Clinical Dementia Rating Scale; FAQ Score $=$ Total score of the Functional Activities Questionnaire.

Table 2: Scores of each patient on cognitive, emotional and functional activities measures before and after PNM stimulation.

\begin{tabular}{|c|c|c|c|c|c|c|}
\hline Measures & \multicolumn{2}{|c|}{ Case P1 } & \multicolumn{2}{c|}{ Case P2 } & \multicolumn{2}{c|}{ Case P3 } \\
\hline & Before & After & Before & After & Before \\
\hline CDR $^{\wedge}$ & 0.5 & 0 & 0.5 & 0.5 & 0.5 & 0 \\
\hline FAQ $^{\wedge}$ & 6 & 1 & 5 & 1 & +1.26 & +0.97 \\
\hline HKLLT $(z)^{\#}$ & -2.17 & +0.45 & +0.40 & +0.71 & -1.23 & +0.09 \\
\hline Rey-O $(z)^{\#}$ & -1.27 & -0.91 & -1.80 & 4 & 3 & 2 \\
\hline CGDS $^{\wedge}$ & 3 & 3 & 6 & 6 & 6 & 3 \\
\hline GAS $^{\wedge}$ & 9 & 4 & 10 & 4 & \\
\hline
\end{tabular}

$\mathrm{CDR}=$ Standard global score of the Clinical Dementia Rating Scale; FAQ = Total score of the Functional Activities Questionnaire; HKLLT $(z)=30$-minute delayed recall performance in the Hong Kong List Learning Test in terms of $z$-score; Rey-O $(z)=30$-minute delayed recall performance in the Rey-Osterrieth Complex Figure Test in terms of z-score; CGDS = Total score of the Chinese Geriatric Depression Scale; GAS

$=$ Total score of the Geriatric Anxiety Scale

${ }^{\wedge}$ Higher scores indicate greater problems / difficulties

\#Positive values indicate better memory performance compared to normative samples 
a normal level after the intervention, as demonstrated by her total score on GAS-10 (reducing from 9 to 4).

Case P2: P2 was a 73-years old female with 13 years of education with a negative history of neurological disorders. At the baseline, she had an above-cutoff adjusted CDRS total score (156.79), suggesting that she did not show signs of dementia, and she scored 0.5 in CDR and 5 in FAQ (Table 1). Besides, her GAS-10 score of 10 suggested a moderate level of anxiety.

Similar to case P1, P2 demonstrated improvement in memory function after the intervention. At the baseline, her performance on the visual memory test was at the $4^{\text {th }}$ percentile rank (-1.8SD; mildly impaired range), and that of the verbal memory was at the $66^{\text {th }}$ percentile rank $(0.4 S D$; average range) (Figure 1). After the intervention, $P 2$ 's visual memory had improved by $1.15 \mathrm{SD}$ from $4^{\text {th }}$ percentile to $26^{\text {th }}$ percentile. The clinical classification had improved from mildly impaired to the low average range. Her verbal memory also improved from $66^{\text {th }}$ percentile to $76^{\text {th }}$ percentile. Her performance was classified in the average range at the baseline and had improved to the high average range after PBM (Figure 1). P2 reported that she experienced some improvement in memory after PBM stimulation. She could recall things after putting some effort towards recalling, while she had failed to do so in the past.

According to the CDR result, she reported moderate difficulty in judgment and problem solving and mild impairment in managing difficult chores and hobbies at the baseline. These functions were reported as normal at the post-intervention assessment. Besides, her FAQ score had reduced from 5 to 1 (Table 2), suggesting that her difficulty experienced in daily functioning became only very minimal. P2 also reported that after PBM she could keep track of current events and manage her financial affairs, for which she had previously required assistance. Besides cognitive improvement, P2 reported being overall happier and having better control of her emotions. Her reduced anxiety was also reflected by a reduction in the GAS-10 score (from 10 to 6), which improved from a moderate level of anxiety to within the normal range (Table 2 ).

Case P3: P3was a 66-years old female with 11-years of education and had a negative history of neurological disorders. At the baseline, she had an above-cutoff adjusted CDRS total score (158.29) and scored 0.5 in CDR and 2 in FAQ (Table 1). She did not show any significant anxiety or depressive symptoms in GAS-10 (6) and CGDS (3).

Her performance on the visual memory test (i.e., Rey-O) was at the $11^{\text {th }}$ percentile, clinically classified in the borderline range. Her verbal memory test performance (HKLLT) was at the $90^{\text {th }}$ percentile (high average level). After the intervention, P3's visual memory improved by $1.32 \mathrm{SD}$ from the $11^{\text {th }}$ percentile to $54^{\text {th }}$ percentile (from borderline to average range), and her verbal memory remained at a high average range $\left(83^{\text {rd }}\right.$ percentile) (Figure 1$)$. She reported that after the intervention, she found it easier to recall her memories; for example, she could remember how to write a Chinese word after thinking for a short period, whereas before PBM stimulation, she could hardly do so.

P3's CDR global score (Table 2) dropped from 0.5 to 0 , and her FAQ score declined from 2 to 0 . Her CDR-Memory rating dropped from "2" (severe memory loss) to "0" (minimal), and CDR-Orientation and CDR-Judgment and Problem Solving rating dropped from "1" (moderate difficulty) to "0" (minimal). In FAQ, she had difficulty remembering appointments and daily events and traveling around at baseline; however, she could generally perform these functions after PBM. Her GAS10 and CGDS scores remained at the same below-cutoff level.

\section{Discussion}

These case reports have shown that patients with $\mathrm{MCl}$ who received PBM stimulation over nine weeks showed improvements in visual and verbal memory function, functional independence, and reduced depressive and anxiety symptoms. All patients well tolerated the PBM treatment, and no side effects were noted. The extent of memory improvement could be as large as a 2.62SD increase on the verbal memory test and a 1.32SD increase on the visual memory test. The mean change of Rey-O scores over the three patients was $+0.94 \mathrm{SD}$, and that of HKLLT scores was $+0.88 \mathrm{SD}$. At baseline, all patients were within the borderline to the moderately impaired range for memory function and had improved up to the normal range after PBM treatment.

There are three $\mathrm{MCl}$ patients of similar ages in our clinic who did not receive PBM, and they did not show such robust improvements after nine weeks without treatment. One patient dropped by 2.3 SD (from average to moderately impaired level) while another remained at the moderately impaired level in Rey-O, and the third remained at the mildly impaired range. In the HKLLT, two patients dropped by 0.62 SD (from borderline to mildly impaired level), and one improved by 0.56 SD. Their mean change in Rey-O and HKLLT scores was both $-0.23 \mathrm{SD}$, which is deterioration. Thus, the improvement observed in the older adults who have received the PBM was not observed in other older adults without receiving the stimulation in our clinic. Therefore, the degree of improvement was probably not due to a practice effect or normal recovery. Besides, natural recovery of memory function impairment within three months for an individual with $\mathrm{MCl}$ is quite unlikely.

It is well known that the amnestic $\mathrm{MCl}$ subtype has a higher conversion rate to dementia [24]. If the PBM treatment could improve the memory function in amnestic $\mathrm{MCl}$, it could prevent $\mathrm{MCl}$ patients from further progression into dementia. However, it should be cautious that our current findings were based on a very small sample size; the actual treatment effect of PBM for $\mathrm{MCl}$ cases remains largely inconclusive. Therefore, further clinical investigations with larger sample sizes and randomized placebo-controlled trials are warranted to verify our pilot findings.

The reduction in depressive and anxiety symptoms of our patients is also noteworthy. Cases P1 and P2 had a history of mood/anxiety disorders; both became happier and less anxious after a few PBM sessions based on their subjective reports and our clinical observations. Such a rapid treatment effect is consistent with previous studies. Schiffer, et al. [25] found significantly reduced depressive and anxiety symptoms in 10 patients with major depression and anxiety disorders after a single four 4-minute PBM treatment. The treatment 
effect was sustained after four weeks. Cassano, et al. [26] also reported that 2 out of 4 depressed patients achieved remission after six PBM treatment sessions. Maiello, et al. [27] found a significant reduction in anxiety symptoms with a large effect size among 12 patients with generalized anxiety disorder after 8-weeks of 20-minutes-per-day self-administered PBM treatment. Nevertheless, the treatment effect of PBM on mood and anxiety problems remains inconclusive and needs further investigation.

\section{Conclusions}

These case reports have provided evidence that 18-sessions of PBM stimulation over nine weeks could significantly improve memory function of individuals with mild cognitive impairment. Besides, their emotional and functional independence levels were also enhanced. The patients well tolerated PBM treatment, and no side effects were noted. These findings suggest that more extensive controlled trials are warranted.

\section{Acknowledgments}

The authors would like to thank Samantha Mui, Kathy Leung, and Quin Chan for collecting and analyzing data.

\section{Funding Support}

This research was funded by the Hong Kong Research Grant Council (14606519).

\section{References}

1. Chan AS, Yeung MK, Lee TL (2019) Can photobiomodulation enhance brain function in older adults? In: Photobiomodulation in the Brain. M Hamblin, YY Huang (edn) Academic Press 427-446.

2. Hamblin MR, Huang YY (eds) (2019) Photobiomodulation in the Brain: Low-Level Laser (Light) Therapy in Neurology and Neuroscience. Academic Press.

3. Moskvin SV, Kochetkov AV (2019) Russian low level laser therapy techniques for brain disorders. In: Photobiomodulation in the Brain. M Hamblin, YY Huang (eds) Academic Press 545-572.

4. Hipskind SG, Grover FL, Fort R, et al. (2018) Pulsed transcranial $\mathrm{red} /$ near-infrared light therapy using light-emitting diodes improves cerebral blood flow and cognitive function in veterans with chronic traumatic brain injury: a case series. Photobiomodul Photomed Laser Surg: 1-8.

5. Naeser MA, Saltmarche A, Krengel MH, et al. (2011) Improved cognitive function after transcranial, light-emitting diode treatments in chronic, traumatic brain injury: two case reports. Photobiomodul Photomed Laser Surg 29: 351-358.

6. Naeser MA, Zafonte R, Krengel $\mathrm{MH}$, et al. (2014) Significant improvements in cognitive performance post-transcranial, red/nearinfrared light-emitting diode treatments in chronic, mild traumatic brain injury: open-protocol study. J Neurotrauma 31: 1008-1017.

7. Hesse S, Werner C, Byhahn M (2015) Transcranial low-level laser therapy may improve alertness and awareness in traumatic brain injured subjects with severe disorders of consciousness: a case series. Int Arch Med 8.

8. Salehpour F, Gholipour-Khalili S, Farajdokht F, et al. (2020) Therapeutic potential of intranasal photobiomodulation therapy for neurological and neuropsychiatric disorders: a narrative review. Rev Neuroscience 31: 269-286.
9. Saltmarche AE, Naeser MA, Ho KF, et al. (2017) Significant improvement in cognition in mild to moderately severe dementia cases treated with transcranial plus intranasal photobiomodulation: case series report. Photobiomodul Photomed Laser Surg 35: 432-441.

10. Chao LL (2019) Effects of home photobiomodulation treatments on cognitive and behavioral function, cerebral perfusion, and resting-state functional connectivity in patients with dementia: a pilot trial. Photobiomodul Photomed Laser Surg 37: 133-141.

11. Berman MH, Halper JP, Nichols TW, et al. (2017) Photobiomodulation with near infrared light helmet in a pilot, placebo controlled clinical trial in dementia patients testing memory and cognition. J Neurol Neurosci 8: 176.

12. Maksimovich IV. Transcatheter intracerebral photobiomodulation in degenerative brain disorders: clinical studies (Part 1). In: Photobiomodulation in the Brain. M Hamblin, YY Huang (eds) Academic Press 515-528.

13. Horner S, Berger L, Gibas K (2020) Nutritional Ketosis and photobiomodulation remediate mitochondria warding off Alzheimer's disease in a diabetic, ApoE4+ patient with mild cognitive impairment: A case report. Photodiagnosis Photodyn Ther 30:101777.

14. Chan AS, Lee TL, Yeung MK, et al. (2019) Photobiomodulation improves the frontal cognitive function of older adults. Int J Geriatr Psychiatry 34: 369-377.

15. Chan AS, Lee TL, Hamblin MR, et al. (2021) Photobiomodulation enhances memory processing in older adults with mild cognitive impairment: a functional near-infrared spectroscopy study. J Alzheimers Dis.

16. Albert MS, DeKosky ST, Dickson D, et al. (2011) The diagnosis of mild cognitive impairment due to Alzheimer's disease: recommendations from the National Institute on AgingAlzheimer's Association workgroups on diagnostic guidelines for Alzheimer's disease. Alzheimer's Dement 7: 270-279.

17. Chan AS, Choi A, Chiu H, et al. (2003) Clinical validity of the Chinese version of Mattis Dementia Rating Scale in differentiating dementia of Alzheimer's type in Hong Kong. J Int Neuropsychol Soc 9: 45-55.

18. Morris JC (1993) The Clinical Dementia Rating (CDR): current version and scoring rules. Neurology 43: 2412-2414.

19. Pfeffer RI, Kurosaki TT, Harrah CH, et al. (1982) Measurement of functional activities in older adults in the community. J Gerontol 37: 323-329.

20. Chan AS (2006) Hong Kong List Learning Test. ( $2^{\text {nd }}$ edn) Hong Kong: Department of Psychology and Integrative Neuropsychological Rehabilitation Center, Chinese University of Hong Kong.

21. Meyers J, Meyers K (1996) Rey Complex Figure and the recognition trial: professional manual. Supplemental norms for children and adolescents. Odessa, Fla: Psychological Assessment Resources.

22. Mueller AE, Segal DL, Gavett B, et al. (2014) Geriatric Anxiety Scale: item response theory analysis, differential item functioning, and creation of a ten-item short form (GAS-10). Int Psychogeriatr 27: 1-13.

23. Chiu HF, Lee HC, Wing YK, et al. (1994) Reliability, validity and structure of the Chinese Geriatric Depression Scale in a Hong Kong context: a preliminary report. Singapore Med J 35: 477480. 
24. Manly JJ, Bell-McGinty S, Tang MX, et al. (2005) Implementing diagnostic criteria and estimating frequency of mild cognitive impairment in an urban community. Arch Neurol 62: 1739-1746.

25. Schiffer F, Johnston AL, Ravichandran C, et al. (2009) Psychological benefits 2 and 4 weeks after a single treatment with near infrared light to the forehead: a pilot study of 10 patients with major depression and anxiety. Behav Brain Funct 5: 46.
26. Cassano P, Cusin C, Mischoulon D, et al. (2015) Near-infrared transcranial radiation for major depressive disorder: proof of concept study. Psychiatry J 2015:352979.

27. Maiello M, Losiewicz OM, Bui E, et al. (2019) Transcranial photobiomodulation with near-infrared light for generalized anxiety disorder: a pilot study. Photobiomodul Photomed Laser Surg 37: 644-650. 